\title{
Enhanced/synthetic vision and head-worn display technologies for terminal maneuvering area NextGen operations
}

\author{
Jarvis (Trey) J. Arthur, III ${ }^{a}$, Lawrence J. Prinzel, III ${ }^{a}$, Steven P. Williams ${ }^{a}$, Randall E. \\ Bailey $^{a}$, Kevin J. Shelton ${ }^{a}$ and R. Mike Norman ${ }^{b}$ \\ ${ }^{a}$ NASA Langley Research Center, Hampton, VA \\ ${ }^{b}$ Boeing Phantom Works, Hampton, VA
}

\begin{abstract}
NASA is researching innovative technologies for the Next Generation Air Transportation System (NextGen) to provide a "Better-Than-Visual" (BTV) capability as adjunct to "Equivalent Visual Operations" (EVO); that is, airport throughputs equivalent to that normally achieved during Visual Flight Rules (VFR) operations rates with equivalent and better safety in all weather and visibility conditions including Instrument Meteorological Conditions (IMC). These new technologies build on proven flight deck systems and leverage synthetic and enhanced vision systems. Two piloted simulation studies were conducted to access the use of a Head-Worn Display (HWD) with head tracking for synthetic and enhanced vision systems concepts. The first experiment evaluated the use a HWD for equivalent visual operations to San Francisco International Airport (airport identifier: KSFO) compared to a visual concept and a head-down display concept. A second experiment evaluated symbology variations under different visibility conditions using a HWD during taxi operations at Chicago O'Hare airport (airport identifier: KORD).

Two experiments were conducted, one in a simulated San Francisco airport (KSFO) approach operation and the other, in simulated Chicago O'Hare surface operations, evaluating enhanced/synthetic vision and head-worn display technologies for NextGen operations. While flying a closely-spaced parallel approach to KSFO, pilots rated the HWD, under low-visibility conditions, equivalent to the out-the-window condition, under unlimited visibility, in terms of situational awareness (SA) and mental workload compared to a head-down enhanced vision system. There were no differences between the 3 display concepts in terms of traffic spacing and distance and the pilot decision-making to land or go-around. For the KORD experiment, the visibility condition was not a factor in pilot's rating of clutter effects from symbology. Several concepts for enhanced implementations of an unlimited field-of-regard BTV concept for low-visibility surface operations were determined to be equivalent in pilot ratings of efficacy and usability.
\end{abstract}

Keywords: Head-worn display, Synthetic Vision, Enhanced Vision, NextGen, Equivalent Visual Operations, Flight Simulation

\section{INTRODUCTION}

Adverse weather accounts for in approximately $70 \%$ of all flight delays in the National Airspace System (NAS). Air traffic operations in the terminal maneuvering area are particularly affected by Instrument Meteorological Conditions (IMC). Estimates posit that airport throughput may drop as much as a factor of 3 during periods of low visibility that can compound into delays throughout the NAS. Existing technologies and new airport construction marginally reduce the "performance gap" for throughput between IMC and Visual Meteorological Conditions (VMC) at the 35 major US commercial hub airports. For example, a new runway at Atlanta (ATL) increased airport throughput by only $3 \% .^{1}$ In any event, for some airports, construction of new runways is not possible and instead must rely solely on technology innovation to reduce the performance gap.

Further author information: Trey Arthur, E-mail: Trey.Arthur@nasa.gov, Telephone: 1-757-864-6609 
The Vehicle Systems and Safety Technologies (VSST) project, under NASAs Aviation Safety Program, is conducting research, development, test and evaluation of crew-vehicle interface technologies to ensure effective crew-system interactions and decisions in all conditions (operational and environmental). One goal is to proactively overcome aircraft safety barriers that might otherwise constrain the full realization of the Next Generation Air Transportation System (NextGen). Part of this research effort involves the use of synthetic and enhanced vision systems and advanced display media as enabling crew-vehicle interface technologies to meet these safety challenges.

One emerging NextGen concept termed, "Equivalent Visual Operations" (EVO), can be achieved using an electronic means to provide sufficient visibility of the external world and other required flight references on flight deck displays that enable the safety, operational tempos, and VMC-like procedures for all weather conditions. Synthetic Vision (SV) and Enhanced Vision System (EVS) technologies are critical enabling technologies to EVO with the ultimate objective of EVO, being one component of a "Better-Than-Visual" (BTV) operational capability; replicating the capacity of today's VMC flight and more importantly, meeting and improving on the safety of today's VMC flight in all-weather NextGen operations.

Synthetic and Enhanced Vision (S/EV) technologies are emerging as standard equipage on today's flight deck. ${ }^{2}$ S/EV generates intuitive visual references for the flight crew/pilot to fly the aircraft as if in VMC independent of the actual visibility or lighting conditions. NASA research aims to extend the present-day $\mathrm{S} / \mathrm{EV}$ concepts to enable VMC-like operational tempos and maintain and improve the safety of VMC while using VMC-like procedures in all-weather conditions. To meet this potential, research is focused on S/EV technology development and human-in-the-loop performance to enable a "visual" approach, landing, roll-out, and surface operations down to $300 \mathrm{ft}$ actual Runway Visual Range (RVR). This BTV operational concept suggests that the minimum aviation system performance standard for BTV technologies should be that as defined by human performance in the same operation using windows during today's VMC operations. Significant research is required to quantify this hypothesized performance standard, and more importantly, to determine if it is indeed an appropriate and sufficient standard for BTV. S/EV work includes the development of fusion methods for synthetic and enhanced vision systems; feature extraction by use of real-time imaging sensors; onboard navigational, sensor, and database integrity monitoring; and appropriate display methods for Head-Up Displays (HUDs) and Head-Worn Displays (HWDs), primary flight and navigation displays, and electronic flight bags.

\subsection{Head-worn display for commercial operations}

NASA has conducted numerous studies evaluating the potential benefits of using HWDs for surface operations. ${ }^{3-5}$ HWDs are small, light weight display devices that can be worn on the head without significant encumbrance (Fig. 1). By coupling the HWD with a head tracker, unlimited field-of-regard can be realized. For a SV-generated scene, the SV-viewpoint position and orientation can be defined via software; thus, an unlimited field-of-regard is achieved since the Synthetic Vision scene is viewable from any virtual camera angle. For sensor imagery, multiple tiled sensors or a "turreted" sensor would be required to realize a large field-of-regard. These implementations have found merits in military avionic applications. ${ }^{6}$

\subsection{Airborne operations}

The BTV operational concept expands on procedural and technological innovations that are being developed to support NextGen operations, such as Flight deck Interval Management (FIM). FIM leverages advancements in communications, surveillance, and navigation to enable flight crews to precisely space their aircraft relative to another aircraft in the terminal maneuvering area. The goal is to improve airport throughput and reduce delays. Using FIM, the air traffic controller instructs the participating aircraft to achieve an assigned interarrival spacing interval at the runway threshold, relative to another aircraft, using on-board automation. The flight crew then takes responsibility to actively fly the FIM operation but the Air Navigation Services Provider (ANSP) retains the responsibility for aircraft separation. NASA research has demonstrated the efficacy of the concept and system-wide and algorithm effects. ${ }^{7}$

Research has recently been completed showing the synergistic potential of combining FIM and S/EV technologies, broaching the concept of BTV. ${ }^{8}$ Simulation testing showed the ability of flight crews to self-separate, 


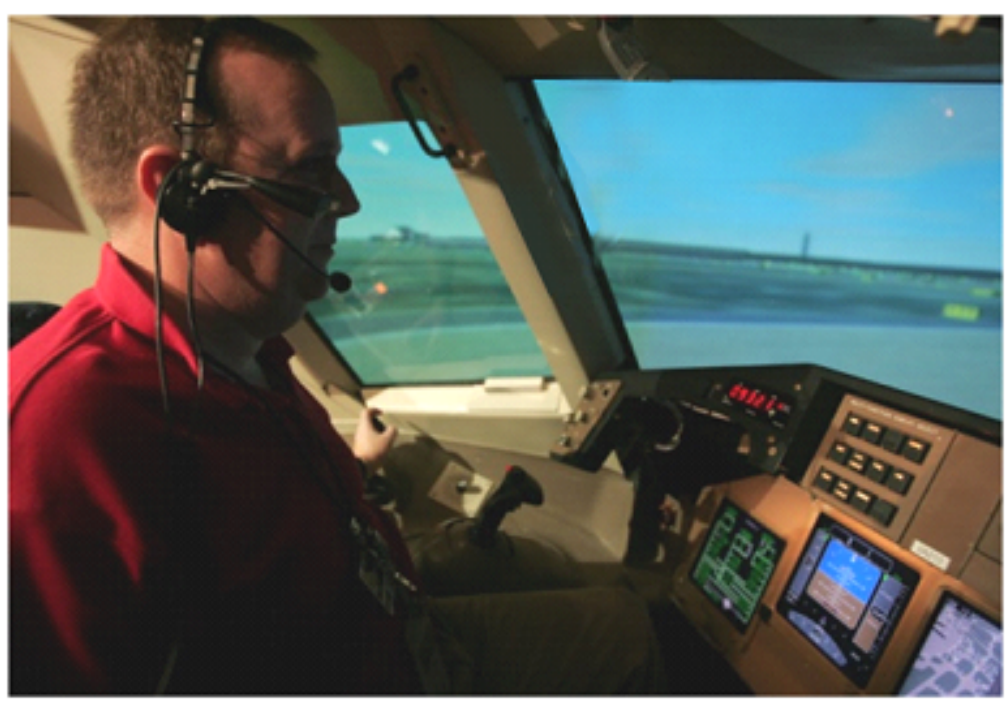

Figure 1. The HWD system integrated with an aviation headset.

wherein the pilot/flight crew accepted responsibility for separation from the designated paired aircraft, and maintained an "equivalent visual contact" through the use of ADS-B and S/EV technologies. Spacing intervals during self-separation approaches followed VMC-like operational profiles while maintaining a very high degree of flight precision, stabilized approach procedures, and excellent traffic/situation awareness. Further, ego- and exo-centric display concepts for terrain, traffic, and airport surface conditions kept the flight crew proactive in the operation and allowed them to easily manage the arrival, through landing (in simulated 700 RVR visibility conditions), roll-out, and turn-off with acceptable workload and sufficient spare attention/workload capacity to easily react to non-normal events that were intentionally staged in the experiment.

Based on the success and efficacy of BTV for in-trail delegated separation and operational credit for lowered visibility landing minimums, the research has now turned to other operations that represent significant challenges for emerging NextGen operational concepts. A significant hurdle to overcoming capacity constraints are simultaneous dependent parallel runway operations at runways separated by less than 4300 feet. In particular, very closely spaced parallel runways have equipment and procedural requirements which significantly limit their use in degraded visual conditions. For example, at San Francisco International Airport (airport identifier: KSFO), Runways 28L and 28R are separated by only 750 feet and the Simultaneous Offset Instrument Approach (SOIA) procedure has been created to maintain airport throughputs. However, at least 4 miles visibility and a 1200 foot ceiling is required - just slightly less visibility conditions than Visual Flight Rules (VFR) operation. When the weather drops below these minima, the airport is reduced to single runway operations substantially reducing arrival rates from 65 to 30 per hour.

KSFO operations are not atypical. To meet the "weather-independence" goal of NextGen, game-changing technology is needed to safely overcome these constraints. One goal of the research described herein is to conduct an initial evaluation of the use of the BTV concept, in concert with the FIM technologies, for very closely spaced simultaneous dependent parallel runway operations in IMC. To achieve the goal, a preliminary analysis was undertaken to assess the use and comparison of the KSFO SOIA under both simulated visual (better than current visibility minimums) and instrument conditions (simulated CAT I) using (a) "visual" out-the-window only or "equivalent visual" with either a (b) head-down or on a (c) head-worn display with synthetic and enhanced vision systems.

\subsection{Surface operations}

NASA has performed previous surface operations research using head-down displays, HUDs and HWDs. ${ }^{5}$ This research has explored numerous operating paradigms and technologies, including the benefits of various display types versus paper charts, binocular versus monocular HWDs, ${ }^{9}$ and flight data presentation (symbology) on the 
display. One goal of NASA's research is to evaluate the efficacy and utility of HWD concepts to support NextGen surface operations.

This study described herein is an extension of the head-up symbology research by exploring two augmented reality concepts which significantly modulate the HWD clutter or occlusion characteristics. HWDs have been shown to uniquely provide the display capabilities desired for piloted surface operations; that is, unlimited fieldof-regard, head-up operations. The design challenge (and certification challenge) is to create this capability without pilot workload, encumbrance, or obscuration of their normal vision.

From previous experiments, pilot comments have highlighted several possible methods to reduce the "clutter" of the overall HWD symbology. First, pilots suggested that the HWD symbology provide more augmented reality rather than virtual reality presentation. Pilots commented that an augmented reality concept is preferred over a virtual reality concept because if an object is visible in the real world, it does not need to be shown virtually on the HWD. Further, pilots have stressed that symbology content in the HWD should only be critical information. The rationale is to de-clutter the HWD by not obscuring visible objects ('if I can see it, I don't need to show a computer-generated representation on top of the visible object').

Figure 2 shows an augmented reality concept developed by NASA to reduce HWD clutter for surface operations. The boxed area containing symbology and the virtual airport represents the view as it would be rendered on the HWD. Outside the boxed area represents the visibility of the actual airport environment with natural vision. In this example, the figure represents a reported visibility of $700 \mathrm{RVR}$. The augmented reality concept draws the S/EV imagery (i.e. the 'virtual airport environment') beyond the reported 700 RVR, a so called "Beyond-RVR" representation. Note that the SV airport is not shown in the HWD up to 700 RVR as this portion of the actual airport can be seen with the unaided eye. Essentially, the SV airport was culled up to 700 RVR (approximately the lower sixth of the display in Fig. 2) to allow pilots to view the actual airport environment. The cleared route (shown as the magenta ribbon) was drawn on top of the yellow taxiway centerline to denote the cleared path. The cleared path always overlays the taxiway centerline if in view on the HWD as it is critical information for runway and taxiway incursion prevention. ${ }^{10,11}$

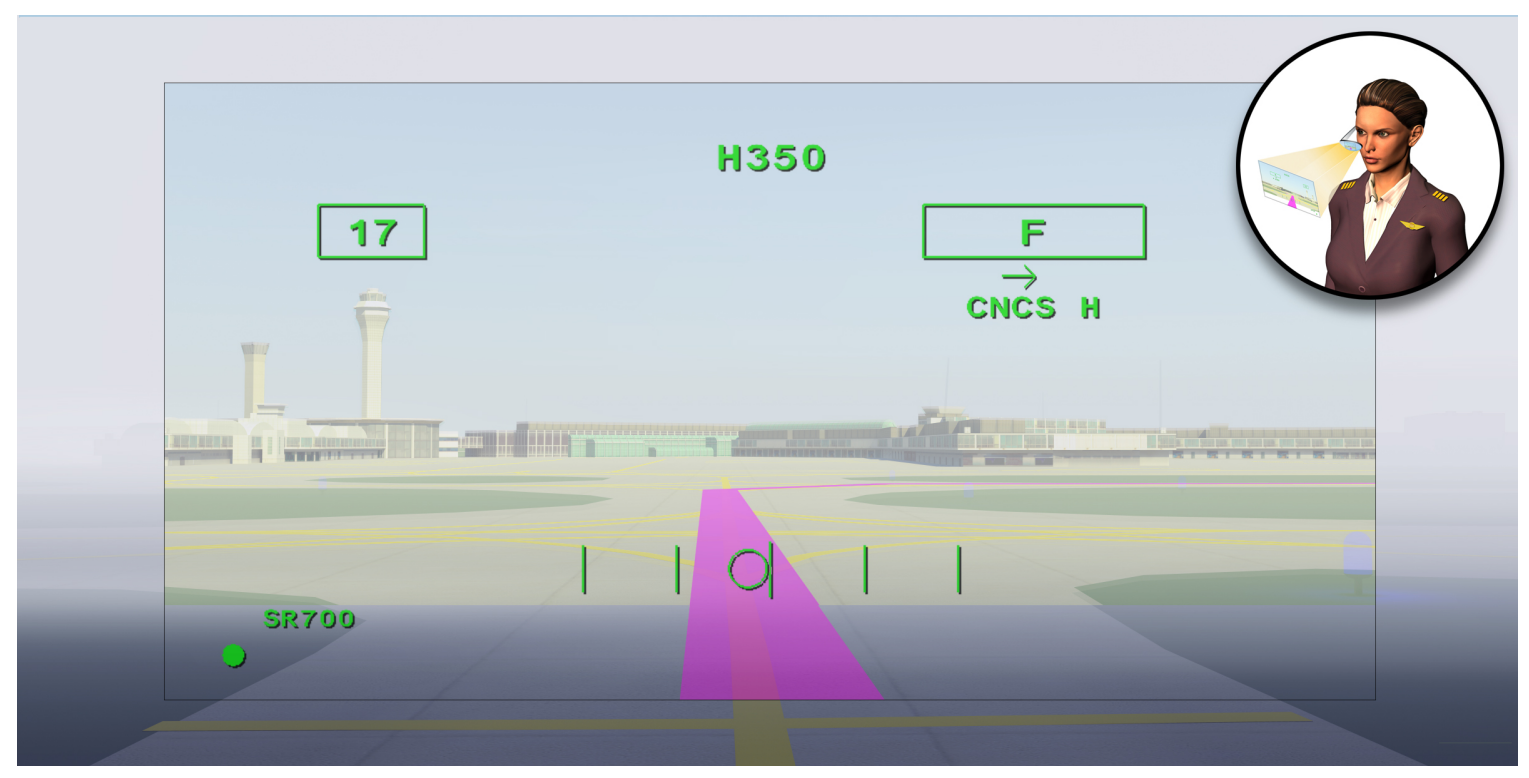

Figure 2. The HWD symbology concept for surface operations.

A second augmented reality symbology technique was also examined. In previous experiments, ${ }^{5}$ generic aircraft models were used to denote traffic on the scene rendered in the HWD. The concept used a variable-size, based on range, unfilled diamond shape rendered at the reported traffic location. This unfilled diamond shape has the advantage of a low clutter "footprint" but lacks traffic heading information or other information possibly derived by using an aircraft model depiction. 
This study examined these 2 augmented reality techniques (Beyond-RVR and traffic rendering) under 3 visibility conditions. The first augmented technique modulated the amount of SV airport environment that was rendered in the HWD. This was compared to a S/EV-generated airport environment that was rendered across the entire HWD field-of-view (used in previous experiments). The second augmented reality technique modulated the way other aircraft were rendered in the HWD. Traffic was rendered as symbology on the HWD as either a generic model aircraft or an unfilled diamond.

\section{EXPERIMENTS}

Two experiments were conducted to determine the efficacy of using a head tracked HWD to explore equivalent visual operations for a closely spaced parallel approach and to explore augmented reality techniques for surface operations. The experiments were conducted back-to-back using the same HWD, simulation facility and Evaluation Pilots (EPs).

\subsection{Head-worn display}

The HWD used in both experiments was a prototype Rockwell Collins ProView ${ }^{\mathrm{TM}}$ SO35-MTV ${ }^{12}$ (Fig. 3). This display was a transparent 800x600 pixel, monochrome green display. The field-of-view (FOV) of the display was measured to be $28.6^{\circ} \mathrm{H}$ by $21.7^{\circ} \mathrm{V}$. Coupled to this display was a prototype head tracker by Intersense, Inc., based on the IS-1200 tracker. The head tracker system used a hybrid combination of optical and inertial sensors. Both the display and tracker were mounted to clear lens glasses. The clear lens glasses were large enough to accommodate most eye wear. The head-borne weight of the combined display and tracker system was approximately 180 grams.

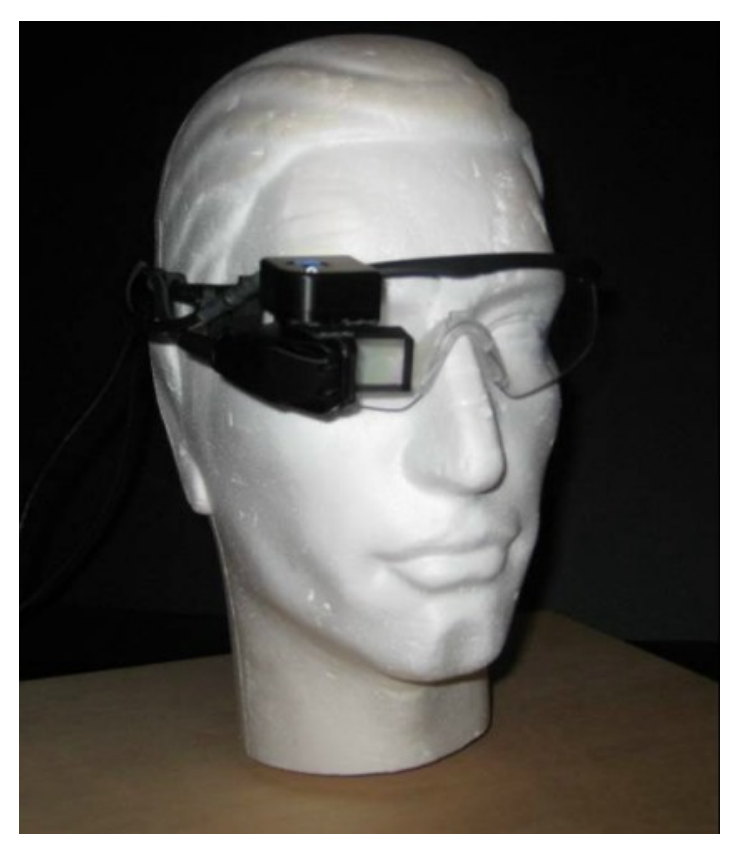

Figure 3. The HWD system.

\subsection{Simulation facility}

Both studies were conducted in the Visual Imaging Simulator for Transport Aircraft Systems (VISTAS) III simulator (Fig. 4) at NASA Langley Research Center (LaRC). VISTAS III is a single pilot fixed-based simulator consisting of a 144 degree by 30 degree out the window visual, large field head-down display (HDD) and pilot input controls. The simulated aircraft in VISTAS III was a large commercial transport type aircraft. 

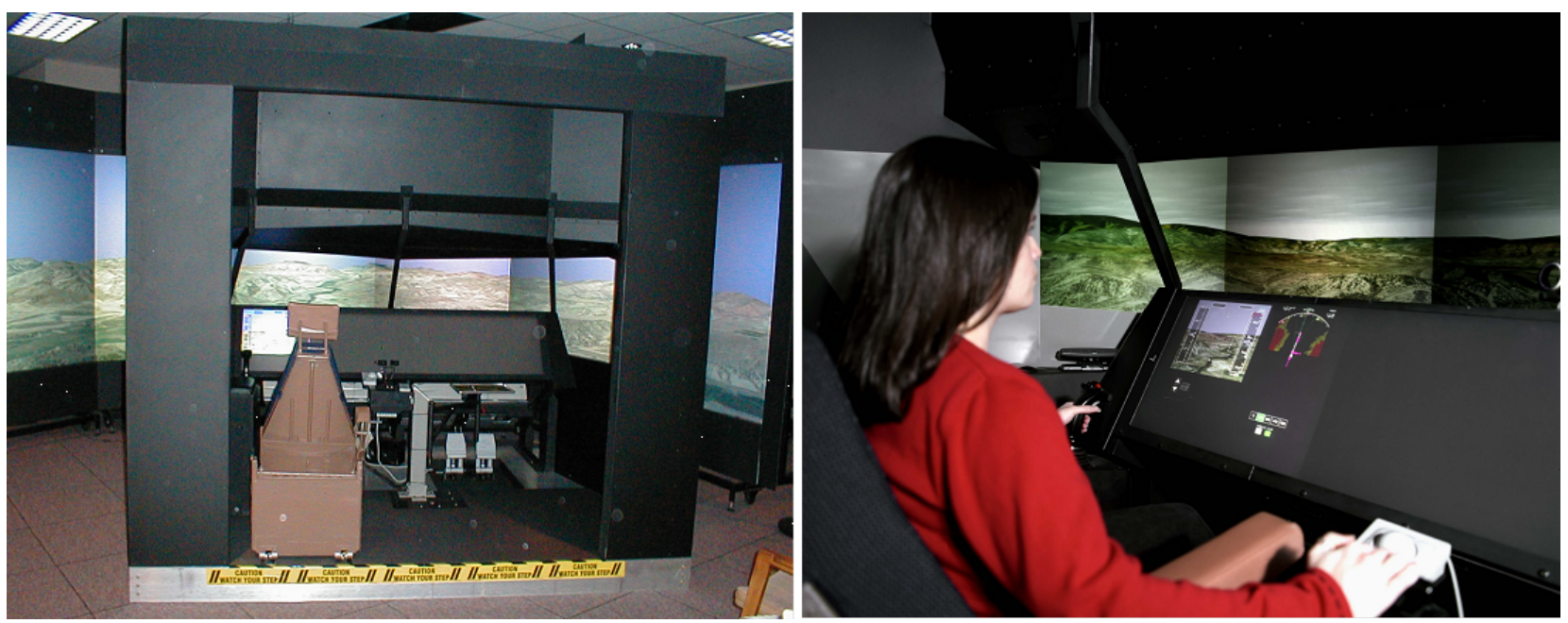

Figure 4. VISTAS III simulator.

\subsection{Evaluation pilots}

Nine commercial EPs from 5 different US airlines participated in both experiments. Five EPs were current or recently retired captains and 4 EPs were current first officers.

The EPs were given a 45-minute briefing to explain the display concepts and the evaluation tasks for both experiments. After the briefing, a 30-minute training session was conducted to familiarize the EPs with the airborne portion of the experiment. EPs were also shown a 10 minute video describing the current KSFO SOIA procedure. Following this training, 1.5 hours of data collection was conducted. After the airborne data collection, a 1-hour training session was conducted to familiarize the EPs with the surface operations portion of the experiment. Following the surface training, data collection trials lasted 1.5 hours. The total time for an EP was approximately 6 hours.

\section{EXPERIMENT ONE: AIRBORNE OPERATIONS}

\subsection{Methodology}

The potential application of BTV technology was explored using simulated KSFO operations. The experiment evaluated the potential of vision-based technology to overcome safety barriers in dependent closely spaced parallel runway operations. To determine initial feasibility, this research evaluated the capability of BTV technologies to enable "equivalent visual" parallel runway operations in IMC. The SOIA at KSFO was chosen as the scenario test case.

\subsubsection{Evaluation task}

Each EP flew as the monitoring pilot while conducting the Localizer Type Directional Aid (LDA) Precision Runway Monitor (PRM) 28R approach to KSFO. Parallel traffic flew the ILS PRM 28L approach. The own-ship aircraft (large commercial transport) was flown using the autopilot coupled to the approach to Runway $28 \mathrm{R}$. The operation was also paired using FIM with traffic approximately 2 minutes ahead. Automated audio call-outs typically made by a first officer were provided to provide awareness to the aircraft configuration. Other simulated audio events included Air Traffic Control (ATC) instructions, traffic party line, and aircraft noises (engines, flap servos, landing gear deployment).

The SOIA requires visual contact with both the airport environment and parallel traffic on $28 \mathrm{~L}$ by the Decision Altitude (DA). Prior to the DA, ATC requested own-ship to respond as to whether the parallel traffic was in sight. EPs were instructed to answer ATC with "equivalent visual contact" if they could see the parallel traffic in either the out-the-window scene or the simulated Forward Looking Infrared (FLIR) sensor image. Note that traffic symbology rendered from ADS-B data was not considered equivalent visual contact. After EPs made 
the equivalent visual contact call, ATC cleared the EPs to land if the parallel traffic was still in sight. If the EP still had equivalent visual contact with the parallel traffic, the EP would respond verbally by saying "landing". If equivalent visual contact was lost, EPs were instructed to call "go-around." All data trials ended at the DA.

\subsubsection{Experiment conditions}

The experiment consisted of three display conditions.

1. Condition A: A variable field-of-regard FLIR presentation (i.e., the FLIR field-of-regard could be panned by the pilot in $15^{\circ}$ increments, azimuth-only) shown on a head-down display.

2. Condition B: A HWD concept which shows a conformal, full field-or-regard FLIR presentation with conformal display of reported traffic, including the FIM-paired traffic.

3. Condition C: A day-time visual approach without S/EV displayed.

A total of 7 data trials were conducted per EP. Each display condition (A through C) was run twice for each EP for a total of 6 nominal runs. The seventh and final data trial was a staged off-nominal event that was not announced; however, during the briefing, it was stressed that a missed approach must be executed if equivalent visual contact with the parallel traffic was lost at or beyond the DA.

The off-nominal scenario represents a case in which the parallel aircraft, while visible during the majority of the data trial, was not visible in the FLIR or with natural vision at the DA. The KSFO SOIA requires that the pilots flying the approach visually acquire the parallel traffic approaching $28 \mathrm{~L}$ and the runway environment no lower than the DA. For the off-nominal condition, the proper response by the EP at the DA was to initiate a go-around and contact ATC.

\subsection{Experiment protocol}

All data reported is subjective. Numerous questionnaire data were completed by the EPs in a semi-structured process, both post-run and post-test. The questionnaires were designed to identify traffic awareness, workload, and approach situational awareness (SA).

Post-run, EPs were asked to rate the following statements on a Likert-type scale (1=low; 10=high).

1. Please rate the amount of overall traffic situation awareness experienced during the trial.

2. Please rate the traffic situation awareness for the parallel traffic during the trial.

3. Please rate the traffic situation awareness for the in-trail traffic during the trial.

4. Please rate the amount of mental workload experienced during the trial.

5. Please rate the ease of making the decision whether to continue the approach at DARNE.

6. Please rate the situation awareness of in-trail spacing during the trial.

Post-run, a Situational awareness rating technique (SART) ${ }^{13}$ was used to assess overall SA for the run. After each data trial, EPs were asked to mark on a scale labeled from low to high their assessment (for that particular data trial) of 3 constructs: 1) Demand on attentional resources; 2) Supply of attentional resources, and 3) Understanding.

Post-test, paired comparison questionnaires were administered to assess the constructs of 1) traffic situation awareness, 2) mental workload and 3) the decision to land. ${ }^{14,15}$ For each of these 3 constructs, EPs compared the 3 display conditions: 1) head-down FLIR, 2) HWD and 3) Visual. 


\subsection{Displays}

A simulated FLIR image was presented to the EP either on a head-down FLIR display or on a HWD (Fig. 5). The FOV of the head-down FLIR display was the same as the HWD FOV. The pan control for the FLIR camera (for those conditions) was pilot-controllable via a button pad. The nominal FLIR simulated EVS-visibility which met or exceeded the visibility required for the approach (i.e., greater than 4 miles).

The HWD condition had the same symbology as the head down FLIR display. The symbology consisted of a velocity vector, counter pointers for airspeed and altitude as defined by MIL-STD-1787B ${ }^{16}$ and a target locator box. The counter pointers were screen-referenced and the velocity vector and target locator box were earth-referenced. The target locator box was driven by simulated ADS-B and showed the traffic call sign on top, the vertical trend via an arrow and vertical speed in feet per minute.

The head-down display for all concepts consisted of a Primary Flight Display (PFD), Navigational Display (ND) with Cockpit Display of Traffic Information (CDTI), Vertical Situation Display (VSD) and an Engine Indicating and Crew Alerting System (EICAS) display (Fig. 6). The PFD was rendered on a 21-inch diagonal LCD with an aspect ratio of 4:3 and the ND was displayed on a 17-inch diagonal LCD with an aspect ratio of 5:4. The Head-down FLIR was rendered on a 15-inch LCD with an aspect ratio of 4:3 with no minification. The PFD was enhanced with SV for all concepts except for the visual condition. For the data trials with the visual condition, the PFD was a typical attitude indicator (blue over brown) with no terrain information. The vertical FOV of the PFD was $40^{\circ}$ and the minification factor of the SV-PFD was approximately 1.4. The ND range was not controllable.

The PFD also had FIM symbology which represented the commanded spacing speed for the paired in-trail aircraft. The ND showed the paired in-trail traffic as a highlighted chevron. The same in-trail traffic was shown on the VSD. Positional inaccuracies were added to the simulated ADS-B information.
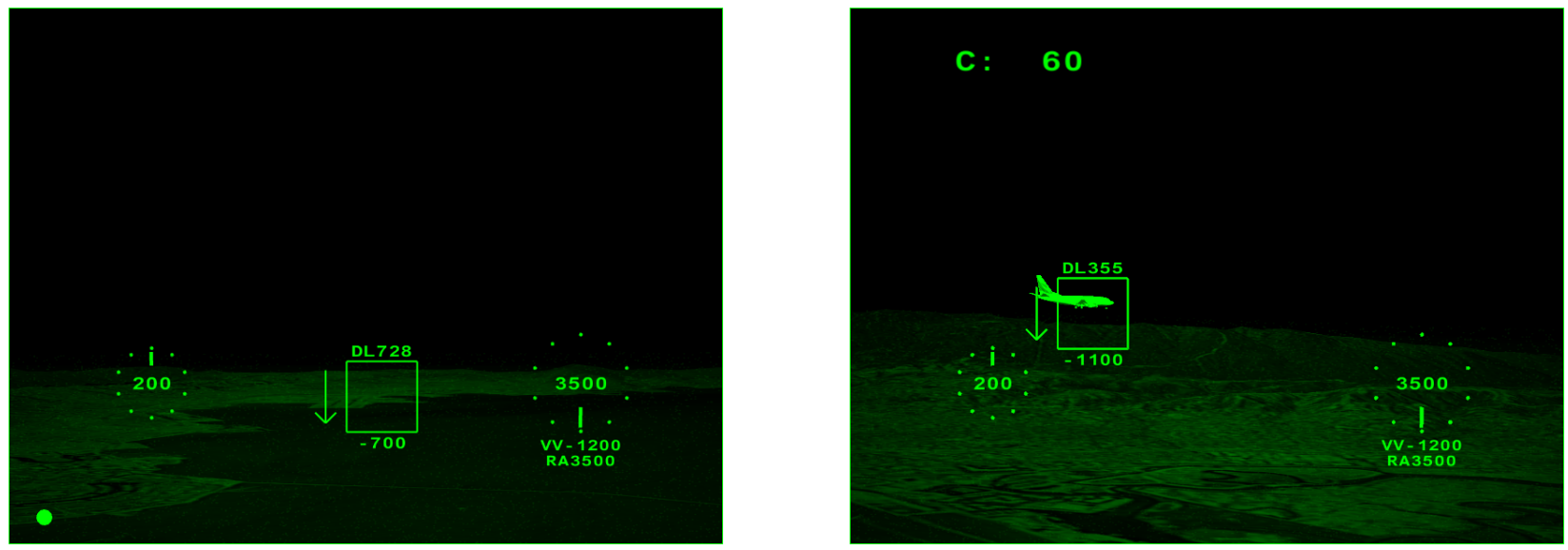

Figure 5. The HWD (left) looking straight ahead on approach to SFO showing the in-trail traffic. The FLIR head-down display (right) concept with the FLIR camera panned $60^{\circ}$ (as denoted by the text "C: 60 " in the upper left corner of the display) to the left showing the parallel traffic.

\subsection{Results}

\subsubsection{Traffic and situation awareness}

Table 1 shows the means and standard deviations (in parentheses) for the subjective measures.

Post-run, awareness of the in-trail and parallel traffic was assessed using Likert-type scales $(1=$ low; $10=$ high $)$. An Analysis of Variance (ANOVA) revealed a significant main effect for measures for in-trail traffic SA, $F(2,17)=$ $3.65, p<0.05$; and parallel traffic SA, $F(2,17)=4.03, p<0.05$. The HWD condition was rated to provide equivalent awareness to the visual condition and both were rated significantly better than the head-down display condition. 

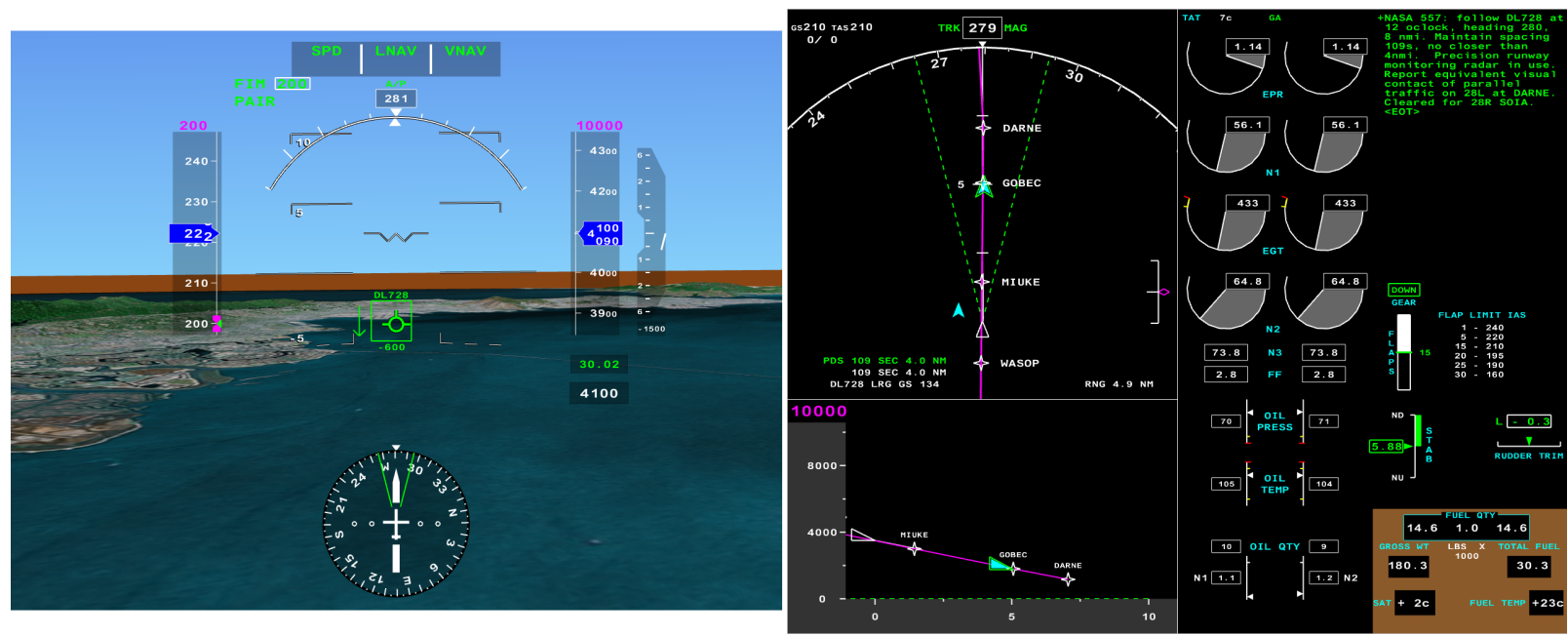

Figure 6. The PFD (left) and the ND/EICAS (right) head-down displays.

Post-run, awareness of the FIM spacing interval and distance was assessed using Likert-type scales (1=low; $10=$ high). An ANOVA was also conducted and there were no significant differences found between the display conditions, $p>0.05$. Overall, pilots rated the display concepts very high for SA of distance interval and conformance maintenance and adherence with time-based ATC FIM clearance.

An ANOVA was conducted on the SART measure. Similar to the results found for the questionnaire measures for traffic SA, there was a significant main effect for the SART, $F(2,17)=4.22, p<0.05$. The HWD condition was not significantly different from the visual condition but both were significantly different from the head-down display condition.

Post-test, the Situation Awareness Subjective Workload Dominance (SA-SWORD) technique ${ }^{14}$ confirmed the SA observed post-run, $F(2,8)=4.75, p<0.05$.

\subsubsection{Mental workload}

Post-run, mental workload during the data run was self-assessed using a Likert-type scale (1=low; $10=$ high). An ANOVA showed a significant main effect for mental workload across display conditions, $F(2,17)=3.87, p<0.05$. EPs rated the workload using the HWD condition the same as the workload for the visual condition; but, the HWD condition was rated lower than the head-down display condition.

Post-test, the Subjective Workload Dominance (SWORD) technique ${ }^{15}$ confirmed the mental workload postrun assessments, $F(2,8)=5.01, p<0.05$.

Table 1. The means and standard deviations for the situation awareness measures.

Display Condition

\begin{tabular}{rlll}
\hline Dependent Measure & Visual & HWD & Head-down Display \\
Traffic Situation Awareness, post-run & $8.5(0.65)$ & $8.5(0.55)$ & $7.0(1.25)$ \\
SART, post-run & $110(12.0)$ & $108(15.0)$ & $85(9.5)$ \\
SA-SWORD, post-test & $0.956(0.011)$ & $0.987(0.020)$ & $0.765(0.041)$ \\
Mental Workload Questionnaire, post-run & $4.5(0.5)$ & $5.0(0.90)$ & $7.0(2.0)$ \\
SWORD (reversed scored), post-test & $0.957(0.12)$ & $0.910(0.03)$ & $0.652(0.17)$ \\
Landing Decision, post-run & $8.5(1.25)$ & $9.0(0.25)$ & $9.0(0.25)$ \\
Landing Decision Paired Comparison, post-test & $0.875(0.15)$ & $0.850(0.10)$ & $0.850(0.10)$ \\
\hline
\end{tabular}




\subsubsection{Decision to land, approach SA}

Post-run, the EPs were asked to rate the ease of making their decision to land using Likert-type scales (1=difficult; $10=$ easy). An ANOVA did not reveal any significant differences in terms of decision-making to continue the approach or go-around at the missed approach point (DA) between the three conditions for either the questionnaire or paired comparison measures, $p>0.05$.

\subsection{Discussion of results}

EPs commented they felt comfortable with the equivalent visual concept generally, and it provided the necessary SA to make a landing decision, at least for this task. The HWD condition was a similar task to the visual condition in that it required EPs to look left to acquire the parallel traffic visually, owing largely to the unlimited field-of-regard and conformality of the HWD which emulates closely to a pilot's natural vision.

The results showed that EPs rated the HWD and the visual condition the same in terms of SA and mental workload. This particular task required looking almost $90^{\circ}$ to the left in order to monitor the parallel traffic. EPs commented that using the HWD used the same scanning motion as with the visual condition since the head tracker simulated the panning of the FLIR camera. Some EPs commented that they were able to detect the traffic easier with the FLIR though this may be a result of this particular simulation environment setup. For this simulation, the thermal contrast of the traffic was high compared to the visual scene used in this study although realistic given the simulated weather and proximity of traffic. In real world conditions, the contrast could possibly be poor in the FLIR image compared to the actual visuals. Conversely, real-world weather conditions, even under $\mathrm{VMC}$, can result in poor acquisition and maintenance of visual contact with traffic using natural vision (e.g., a reason for the development of CDTI-assisted visual separation concepts). Therefore, the characteristics of the weather are important considerations in evaluation of any potential use of enhanced vision systems to support $\mathrm{BTV} / \mathrm{EVO}$ operations.

A few EPs liked the head-down condition as it was close to the PFD and ND; however, the head-down condition was rated as the highest workload and lowest SA. The head-down FLIR presentation could easily be incorporated into the scan pattern without having to look far off to the left. As the head-down task was in IMC, they were already head-down, thus it made monitoring the parallel traffic easier. However, the head-down FLIR required the EP to make constant camera pan adjustments. Also, the scenario flown did not have any turbulence. Some EPs were concerned that with only pan control, certain pitch and roll combinations would cause the parallel traffic to go outside the vertical FOV, thus requiring the need for pitch control. It was suggested that the FLIR camera auto-track the traffic to reduce workload on the pilot. Additionally, part of the preference for the head-down condition was motivated due to the prototype nature of the HWD implementation. The HWD was not optimized for commercial operational use and garnered some skeptical and negative responses.

All EPs detected the off-nominal event and requested a go-around when visual or equivalent visual contact was lost with the parallel traffic. In the case of the HWD and the head-down conditions, the FLIR image was slowly faded completely out over a 10 -second period for the off-nominal event only. This simulated a situation wherein the parallel traffic enters into a heavy moisture cloud that can not be penetrated by the FLIR sensor. For the visual condition off-nominal event, the 28L parallel traffic slowed down and was being overtaken by ownship, resulting in the traffic not being visible out the window. In all cases, EPs felt they could easily determine whether contact with the parallel traffic was obtained and could be maintained and that the airport environment was in view so they could decide whether to continue the approach passed the missed approach point or call for a go-around as prescribed in the SOIA procedure.

\section{EXPERIMENT TWO: SURFACE OPERATIONS}

\subsection{Methodology}

Prior the start of this experiment, 6 pilots participated in informal taxi sessions to provide feedback regarding the presentation of the SV airport on a HWD for surface operations. Also, their opinions were solicited regarding the display of the cleared taxi route on the HWD. From these informal sessions, pilots indicated their desired brightness level for both the SV airport as well as the cleared route. 


\subsubsection{Evaluation task}

For the surface operations experiment, EPs were tasked to taxi a simulated large commercial transport aircraft from the runway to the gate at Chicago O'Hare (KORD) airport. All taxi routes began with the aircraft stopped on the runway and the active route having been transmitted. This taxi route was displayed as text as well as graphically on the Electronic Moving Map (EMM) and HWD. The taxi route on the HWD was displayed conformally as an augmented reality enhancement of the taxiway centerline. In other words, the computer generated cleared route line was placed on top of the actual taxiway centerline. All of the taxi scenarios were conducted in the daytime under variable visibility/fog conditions. Besides own-ship, all other traffic aircraft were on the ground and stationary.

\subsubsection{Experiment conditions}

The experiment varied 3 factors (described in detail in subsequent sections):

1. Beyond-RVR concept that renders virtual airport objects (such as runways, taxiways, centerlines, tower(s), buildings) obscured by the weather in the HWD. In other words, the HWD virtually displays airport items and traffic that cannot be seen naturally and thus, not obscuring naturally visible objects.

2. RVR visibility value.

3. Traffic rendering style.

\subsubsection{Beyond-RVR condition}

The Beyond-RVR concept included 3 variations:

1. A pilot-controllable condition where the EP was allowed to control the amount (via a knob in the cockpit) of the SV information that was rendered.

2. A fixed condition where the pilot was not allowed to control the amount of the SV airport rendered; the amount of the SV airport rendered was automatically set based on the reported RVR.

3. A total SV environment condition where the Beyond-RVR was disabled; the SV airport information was displayed everywhere (even over visible objects) and was not pilot controllable.

The first 2 items are new concepts and were compared to the previous concept (total SV environment, Item 3 ) that was tested in a previous experiment. ${ }^{5}$

\subsubsection{Visibility condition}

The outside visibility was varied over 3 ranges: 1) 300 RVR, 2) 600 RVR and 3) 2400 RVR.

\subsubsection{Traffic rendering style}

Traffic was rendered on the HWD in 2 different styles: 1) unfilled diamond and 2) generic aircraft model. The unfilled diamond model was a simple representation of an aircraft (using ADS-B symbol set) which showed aircraft position as reported by ADS-B. The second rendering of traffic used a generic aircraft model to depict other aircraft position and heading. This rendering is thought to be more intuitive but at the expense of increased clutter.

\subsection{Experiment design and procedure}

The experiment matrix was a full factorial design of 18 runs: 3 Beyond-RVR display concepts $\times 3$ Visibility RVR $\times 2$ Traffic Rendering. Each run averaged 3 minutes for 1.5 hours of data collection.

The data runs were blocked into 3 groups of 6 runs. For each 6-run block, the visibility condition (300 RVR, 600 RVR and 2400 RVR) was held constant while the Beyond-RVR and traffic rendering condition was varied. Following each 6-run block, a clutter questionnaire was given to the EPs.

At the end of the 18 data runs, EPs were administered 2 SA-SWORD questionnaires. The first SA-SWORD questionnaire asked EPs to compare the 3 Beyond-RVR concepts for each visibility condition. The final SASWORD asked pilots to compare each of the 3 Beyond-RVR concepts regardless of visibility. 


\subsection{Displays}

Figure 7 (left image) shows only the symbology on the HWD. As the HWD is see-through, black regions in the figure would be areas where "real-world" airport objects would be seen. The concept illustrated in Figure 2 shows a better representation of the EPs view in the HWD. However, unlike the color SV imagery shown in Figure 2, the HWD used in the experiment was monochrome green. The symbology was developed from a previous experiment and has not yet been significantly altered based on the results of that experiment. The green ribbon depicts the cleared path and is not affected by the Beyond-RVR concept. It conformally overlays the 'real' yellow painted taxi centerline which allows the pilot to easily follow the route head-up. The SV airport, shown as the KORD tower, concourse buildings and airport surface in Figure 7, is also conformally displayed. All other symbology is screen-referenced. The brightness of the path was set to $80 \%$ opaqueness $(0 \%$ is completely see-through). The SV KORD airport brightness was set to $50 \%$ opaqueness. The text "SR700" indicates that the pilot has selected the SV information to be clipped up to a 700 foot near clipping plane from the pilot eye point, thus the black portion at the bottom of Figure 7 would allow an unobscured view of the real airport environment. Other symbology shown is the ground speed ("17"), the current heading ("H350"), the current taxiway is boxed ("F"), the next route point (left turn, as denoted by the arrow, to "CNCS H") and the path error (i.e., the localizer-type deviation scale and status symbology). The path error scale is detailed on page 42 in the paper by Arthur, et al. ${ }^{5}$ The filled green circle in the bottom left of the display is an indication to the EP that the head tracker is operating nominally.

A head-down EMM display was rendered as shown in Figure 7. This display shows the airport surface from an overhead perspective. The display shows the cleared route, both graphically and textually, taxiway labels and airport environment. The range of the EMM was fixed to a range scale of $0.5 \mathrm{nmi}$.
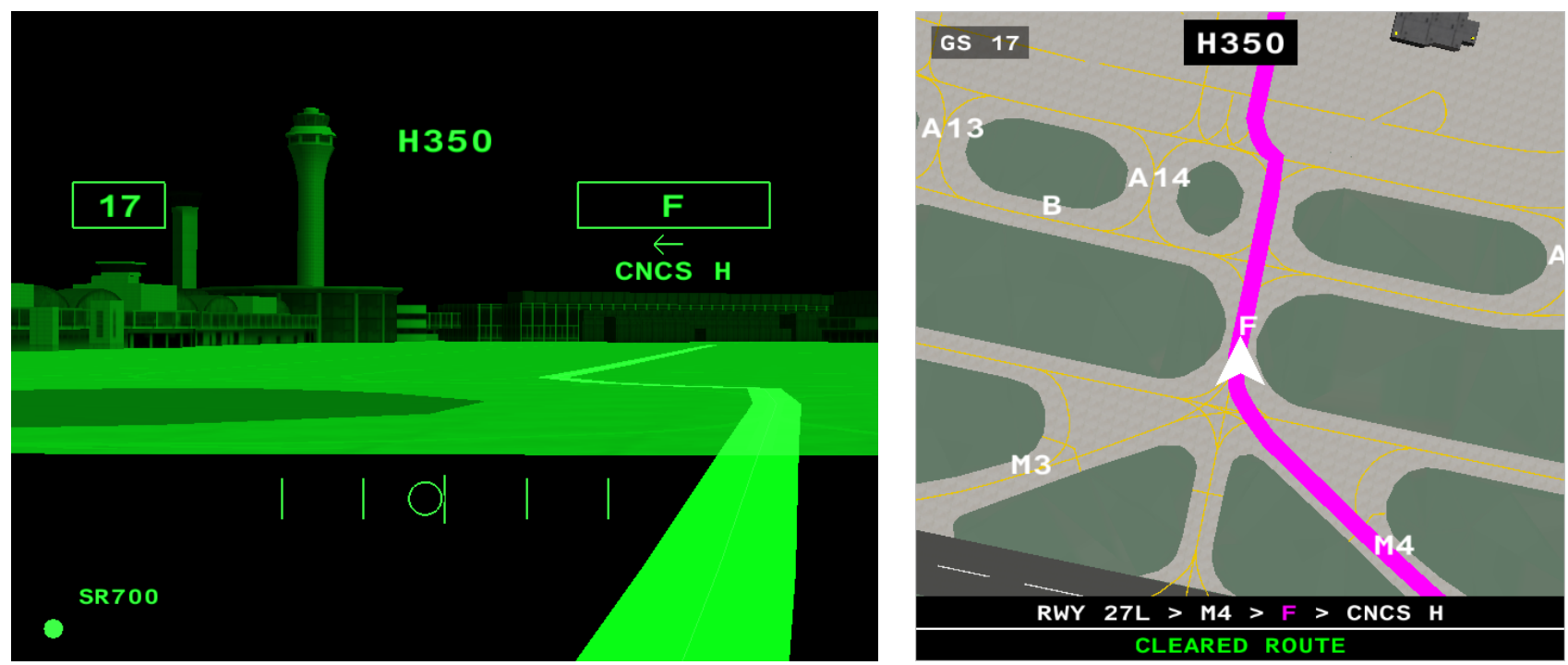

Figure 7. The HWD (left) and the EMM (right) displays. As the HWD is translucent, black regions in the HWD figure represent see-through areas.

\subsection{Results}

\subsubsection{Situation awareness}

Post-test, a SA-SWORD questionnaire was administered that compared the Beyond-RVR conditions with each of the 3 visibility conditions. An ANOVA on the paired comparison for display condition was not significant across either the three displays, or visibility, or interaction of display condition and the visibility, $p>0.05$. Table 2 presents the paired comparison means and standard deviations.

At the end of all data trials, EPs were asked which traffic rendering style they preferred. Eight out of 9 EPs preferred the generic aircraft model over the open diamond symbology. 
Table 2. The paired comparison means and standard deviations.

Display Condition

\begin{tabular}{rlll}
\hline Visibility & Pilot-Controlled & Fixed & Total SV Environment \\
$300 \mathrm{RVR}$ & $0.545(0.15)$ & $0.550(0.10)$ & $0.550(0.10)$ \\
$600 \mathrm{RVR}$ & $0.450(0.20)$ & $0.500(0.50)$ & $0.550(0.03)$ \\
$2400 \mathrm{RVR}$ & $0.265(0.22)$ & $0.301(0.35)$ & $0.285(0.43)$ \\
\hline
\end{tabular}

\subsection{Discussion of results}

All EPs commented the SV airport (i.e., unlimited field-of-regard S/EV information), regardless of the BeyondRVR concept tested, increased their SA. This finding is consistent with the results from previous experiments conducted with HWD for surface operations. ${ }^{5}$ It was hypothesized that either the pilot-controlled or the fixed Beyond-RVR concept would be preferred to the total SV environment concept, but the results showed there were no differences in any of the Beyond-RVR concepts. Since this contradicts comments made from a previous experiment, those EPs who preferred the total SV environment were asked their opinion on the concept. They stated they liked having as much information as possible and at the low visibility conditions, there wasn't much difference between the total SV environment versus a culled Beyond-RVR scene. To compound this further, the KORD airport model used to generate the out-the-window was identical to the perspective S/EV airport view in the HWD. In previous experiments, the airport models rendered in the out-the-window and the HWD differed. Since the airport models were the same in this experiment, there was no obscuration of "real-world" elements by design. If the out-the-window scene contained such elements as taxiway signs which were not part of the S/EV HWD view, it is hypothesized that the Beyond-RVR concepts would be preferred to a total SV environment.

Regarding traffic rendering, EPs preferred the generic aircraft models rather than unfilled diamonds. EPs commented that the airplane model was easy to interpret while the unfilled diamonds lacked heading information. Further, while EPs felt the generic aircraft model was sufficient, some EPs felt that at least 2 models should be used to denote small and large aircraft.

During two data trials, data collection was ended due the EPs reporting simulation sickness symptoms. ${ }^{17}$ Simulator Sickness is a form of motion sickness experienced by pilots in either fixed or motion simulators from large FOV motion. This can be exacerbated by the total system latency inherent in head tracked HWDs. Simulator Sickness is experienced more with helicopter simulators than fixed wing simulators. ${ }^{18}$ This is believed to be caused by large FOV visuals close to the ground with lots of motion (Vection illusion) and the lack of motion cues with coupling visual scene. We conjecture that helicopter motion is similar in many regards to fixed-wing large transport surface operations simulations. During ground turns, pilots must use very large head-motions to clear the turns and then the aircraft makes very large and rapid angular movements, especially in over-steering situations, to make the turns. Surface operations simulator sickness has been reported in previous tests ${ }^{5}$ while no simulator sickness symptoms were reported in these same simulators for fixed-wing flight simulations.

Both EPs who experienced Simulator Sickness reported the cockpit temperature to be "very warm" and had a sense of disorientation. One of these EPs reported having simulator sickness symptoms from previous simulations. As a result of the EPs experiences, the temperature of the room was reduced, the training protocol was changed and the number of data trials was reduced. After these changes were made, simulator sickness symptoms were no longer reported by EPs.

\section{CONCLUSIONS}

A HWD concept, coupled with a head tracker, was used to evaluate full-field-of-regard S/EV concepts for airborne and surface operations. A fixed-based simulation experiment explored the viability of using a HWD for equivalent visual operations during closely spaced parallel operations. In addition, surface operations were conducted to refine symbology concepts.

For the airborne task, pilots rated using a HWD in IMC the same as the visual approach in terms of situation awareness and mental workload. These results are further evidence of the significant potential of 
BTV technologies to enable equivalent visual operations. Prinzel et al. (2011) provided initial evidence on the use of these technologies for delegated in-trail separation for nominal terminal movement area operations. The present study expands this research in showing potential value of these technologies for enabling equivalent visual simultaneous offset instrument approaches. Several simplifying assumptions were used in this initial study and further investigation and development is needed. In particular, the effect of realistic ADS-B sensor models and the development of EVS sensor technologies to meet the minimum performance levels envisioned in this operation must be evaluated. Additional steps would be to continue to evaluate the BTV concepts for other operations that could benefit from reducing or eliminating the visibility requirements to conduct the operations. Such examples include leveraging new approvals to conduct simultaneous dependent staggered straight-in operations to runways separated as close as 1200 feet, Simplified Aircraft-Based Paired Approach Concept (SAPA), and true "visual" approaches in which parallel traffic, to runways separated by less than 2500 feet, fly abeam one another and must maintain "visual" (or "equivalent visual") delegated separation. Other future directions include delegated equivalent visual separation for departures, and continued research on development of BTV concepts for equivalent visual surface operations and integration of these concepts across phases of flight and operational needs.

For the surface operations study, there were no reported differences between the 3 augmented reality concepts or influence of natural visibility or on situation awareness or pilot workload. The pilot comments supported the value of BTV to increase capacity and safety in the airport movement area and that innovative approaches for intuitive display of S/EVS have merit and research should continue. Pilots did comment that they preferred traffic be rendered in the HWD as generic aircraft models rather than iconic (rendered using an open diamond shape).

The encumbrance of the HWD was significantly less that previous NASA studies; however improvement must continue toward reducing the system latency. These results were obtained using a ground simulation facility with no motion effects. Motion simulation should be conducted to tease out the influence of vestibular stimulation and of course, these results must be fully validated during actual operations, focusing on daylight readability (particularly for evaluating the utility and capabilities for color), disorientation and illusion issues (i.e., "simulator sickness"), and augmented reality requirements for symbology and S/EV imagery.

\section{REFERENCES}

1. E. Parker, F. Soloninka, and J. Littleton, "A new approach to measuring the effect of weather on NAS performance," Air Traffic Control 52, No. 3, pp. 7-12, Summer 2010.

2. R. Bailey, L. Prinzel, L. Kramer, and S. Young, "Concept of operations for integrated intelligent flight deck displays and decision support technologies," Tech. Rep. NASA/TM-2011-000000, NASA Langley Research Center, Hampton, VA, February 2011. in press.

3. J. Arthur III, L. Prinzel III, S. Williams, and L. Kramer, "Synthetic vision enhanced surface operations and flight procedures rehearsal tool," in Enhanced and Synthetic Vision Proceedings of SPIE, J. G. Verly and J. J. Guell, eds., 6226, p. 62260I, SPIE, (Bellingham, WA), 2006.

4. J. Arthur III, L. Prinzel III, K. Shelton, R. Bailey, L. Kramer, S. Williams, and R. Norman, "Design and testing of an unlimited field-of-regard synthetic vision head-worn display for commercial aircraft surface operations," in Enhanced and Synthetic Vision 200\%, J. G. Verly and J. J. Guell, eds., 6559, SPIE, (Bellingham, WA), April 2007.

5. J. J. Arthur III, L. Prinzel III, R. Bailey, K. Shelton, S. Williams, L. Kramer, and R. Norman, "Head-worn display concepts for surface operations for commercial aircraft," Tech. Rep. NASA/TP-2008-215321, NASA Langley Research Center, Hampton, VA, June 2008.

6. J. Sanders-Reed, K. Bernier, and J. Guell, "Enhanced and synthetic vision system (ESVS) flight demonstration," in Enhanced and Synthetic Vision 2008, 6957, SPIE, (Orlando, FL), Mar. 2008.

7. B. Barmore, "Airborne precision spacing: A trajectory-based approach to improve terminal area operations," in 9th ATIO Conference, ATIO, (held in Hilton Head, SC), 2009.

8. L. Prinzel, K. Shelton, L. Kramer, J. Arthur, R. Bailey, and K. Elis, "Flight deck-based delegated separation: Evaluation of a merging and spacing system with synthetic and enhanced vision technology," in 2011 Digital Avionics conference, DASC, (Seattle, WA), October 2011. in press. 
9. F. Cupero, B. Valimont, J. Wise, C. Best, and B. D. Mers, "Head worn display system for equivalent visual operations," Tech. Rep. NASA/CR-2009-215781, Hampton, VA, Jul 2009.

10. D. R. Jones and J. M. Rankin, "A system for preventing runway incursions," Journal of Air Traffic Control 44, July - September 2002.

11. D. R. Jones, "Runway incursion prevention system simulation evaluation," in 21th Digital Avionics Systems Conference, October 2002.

12. "Rockwell Collins ProView SO35-MTV." http://rockwellcollinsaviation.org/content/pdf/pdf_11503.pdf.

13. R. M. Taylor, "Situational awareness rating technique (SART): The development of a tool for aircrew systems design," in AGARD Conference Proceedings No 478, Situational Awareness in Aerospace Operations, pp. 3-1 - 3-37, (Aerospace Medical Panel Symposium, Copenhagen), October 1990.

14. M. A. Vidulich and E. R. Hughes, "Testing a subjective metric of situation awareness," in Human Factors Society 35th Annual Meeting, pp. 1307-1311, Human Factors Society, (Santa Monica, CA), 1991.

15. M. A. Vidulich, "The use of judgment matrices in subjective workload assessment - the Subjective WORkload Dominance (SWORD) technique," in Human Factors and Ergonomics Society, 33rd annual meeting, pp. 1406-1410, (Denver, CO), October 1989.

16. United States Air Force, "Aircraft display symbology," Tech. Rep. MIL-STD-1787B, Department of Defense, Wright-Patterson AFB, OH, April 1996.

17. R. S. Kennedy, N. E. Lane, K. S. Berbaum, and M. G. Lilienthal, "Simulator sickness questionnaire: An enhanced method for quantifying simulator sickness," International Journal of Aviation Psychology , pp. 203220, 1993.

18. D. Johnson, "Simulator sickness research summary," Tech. Rep. 1832, U.S. Army Research Institute, April 2005. 